\title{
Antibiotic resistance really starts to hurt
}

\author{
C. Ruef
}

Published online: 9 November 2011

(C) Springer-Verlag 2011

The topic of antibiotic resistance has received wide coverage not only in the scientific medical literature but also in newspapers and other media sources. For most members of the general population, with the possible exception of individuals working in the healthcare sector, information on antibiotic resistance is generally presented in the relatively impersonal form of overall statistics, graphics, percentages, and numbers. These numbers have shown an upward trend in terms of the proportion of resistant bacteria. While most clinicians working in the field of infectious diseases, intensive care, and other healthcare providers in the hospital environment, as well as those working outside of hospitals, albeit less often, have been confronted with patients infected by bacteria exhibiting a broad range of resistances against antibiotics, such experiences are still relatively rare in many countries of the Western world. Thus, although some physicians have witnessed lethal outcomes due to essentially non-treatable infections, the actual problem of antibiotic resistance is considered by many healthcare providers to be primarily an epidemiologic and public health problem. As such, they consider it necessary to curtail inappropriate antibiotic use and implement infection control measures to prevent the spread of these pathogens.

Two studies in this issue of Infection demonstrate that the topic of antibiotic resistance, while still best described using statistics, is beginning to impact individuals outside of the hospital environment. In their article, Meyer and colleagues

C. Ruef $(\square)$

Division of Infectious Diseases and Hospital Epidemiology,

HAL 14C, University Hospital of Zurich,

8091 Zurich, Switzerland

e-mail: christian.ruef@usz.ch report that co-infection and co-colonization has increased steadily over the course of just a few years, as shown in their study on co-colonization involving the co-presence of extended-spectrum beta-lactamases (ESBL)-producing Enterobacteriaceae, vancomycin-resistant Enterococcus faecium and/or methicillin-resistant Staphylococcus aureus (MRSA) [1]. In another study involving patients in California, Abrahamian and colleagues describe the clinical impact of antibiotic resistance on women with acute uncomplicated cystitis [2]. These authors report that clinical cure rates were markedly lower $(<50 \%)$ if the causative Enterobacteriaceae were resistant to trimethoprim/sulfamethoxazole. This resistance resulted in a reduced healthrelated quality of life as a simple consequence of a lower clinical cure rate, which could clearly be explained by the presence of resistance to trimethoprim/sulfamethoxazole.

The presence of pathogenic agents resistant to a specific antibiotic may be considered to be less threatening than the multi-resistance already confronting physicians at hospitals. However, if the former can have such a negative impact on quality of life, as reported in the two articles described in this issue of Infection, what can be expected if the broadness of resistance widens in the near future?

The description of a negative impact on the quality of life in the paper of Abrahamian [2] should serve as a further warning to all healthcare professionals that the time to activate and reinforce our fight against the spread of antibiotic resistance is NOW.

\section{Ruef, Editor-in-Chief, Infection}




\section{Conflict of interest None.}

\section{References}

1. Meyer E, Ziegler R, Mattner F, Schwab F, Gastmeier P, Martin M. Increase of patients co-colonized or co-infected with methicillin- resistant Staphylococcus aureus, vancomycin-resistant Enterococcus faecium or extended-spectrum $\beta$-lactamase-producing Enterobacteriaceae. Infection. 2011;39. doi:10.1007/s15010-0110154-0.

2. Abrahamian F, Krishnadasan A, Mower W, Moran G, Coker J, Talan D. The association of antimicrobial resistance with cure and quality of life among women with acute uncomplicated cystitis. Infection. 2011;39. doi:10.1007/s15010-011-0163-Z. 